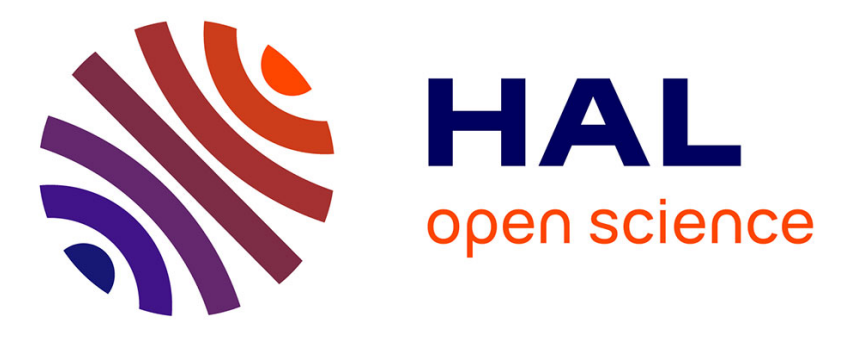

\title{
Impact of Soft Tissue Heterogeneity on Augmented Reality for Liver Surgery
}

Nazim Haouchine, Stephane Cotin, Igor Peterlik, Jeremie Dequidt, Mario

Sanz Lopez, Erwan Kerrien, Marie-Odile Berger

\section{To cite this version:}

Nazim Haouchine, Stephane Cotin, Igor Peterlik, Jeremie Dequidt, Mario Sanz Lopez, et al.. Impact of Soft Tissue Heterogeneity on Augmented Reality for Liver Surgery. IEEE Transactions on Visualization and Computer Graphics, 2015, 21 (5), pp.584 - 597. 10.1109/TVCG.2014.2377772 . hal-01136728

\section{HAL Id: hal-01136728 \\ https://inria.hal.science/hal-01136728}

Submitted on 1 Sep 2015

HAL is a multi-disciplinary open access archive for the deposit and dissemination of scientific research documents, whether they are published or not. The documents may come from teaching and research institutions in France or abroad, or from public or private research centers.
L'archive ouverte pluridisciplinaire HAL, est destinée au dépôt et à la diffusion de documents scientifiques de niveau recherche, publiés ou non, émanant des établissements d'enseignement et de recherche français ou étrangers, des laboratoires publics ou privés. 


\title{
Impact of Soft Tissue Heterogeneity on Augmented Reality for Liver Surgery
}

\author{
Nazim Haouchine, Stephane Cotin, Igor Peterlik, Jeremie Dequidt, \\ Mario Sanz Lopez, Erwan Kerrien and Marie-Odile Berger
}

\begin{abstract}
This paper presents a method for real-time augmented reality of internal liver structures during minimally invasive hepatic surgery. Vessels and tumors computed from pre-operative CT scans can be overlaid onto the laparoscopic view for surgery guidance. Compared to current methods, our method is able to locate the in-depth positions of the tumors based on partial three-dimensional liver tissue motion using a real-time biomechanical model. This model permits to properly handle the motion of internal structures even in the case of anisotropic or heterogeneous tissues, as it is the case for the liver and many anatomical structures. Experimentations conducted on phantom liver permits to measure the accuracy of the augmentation while real-time augmentation on in vivo human liver during real surgery shows the benefits of such an approach for minimally invasive surgery.
\end{abstract}

Index Terms_-Image-guided Simulation, Biomechanical Modeling, Real-Time Augmented Reality, Computer Assisted Surgery.

\section{INTRODUCTION}

$\mathrm{C}$ ONSIDERABLE advances in medicine have seen the emergence of new surgery techniques such as Minimally Invasive Surgery (MIS). In this technique, the surgeon does not interact with organs directly as in the open surgery, but manipulates instruments inserted through trocars placed in small incisions in the wall of the abdominal cavity. The surgeon can observe these instruments on a display showing a video stream captured by an endoscopic camera inserted through the navel. The main advantage of the method is in reduced pain and time recovery. In addition, reducing bleeding and risks of infection have increased the number of operations done by a minimally invasive manner. Although the MIS procedure is considered as a well-established technique, it remains quite complex from the surgical standpoint mainly due to the limited visual feedback and indirect manipulation. On the other hand, the presence of a video-capturing device such a mono or stereo laparoscopic camera has naturally led the research community to investigate the use of Augmented Reality to guide the surgeon during the procedure (see Figure 1). Indeed, internal structures computed from pre-operative scans such as tumors and vessels can be superimposed onto the intra-operative images to facilitate the orientation and navigation during the surgery.

In order to establish a full augmented reality system

- Nazim Haouchine, Stephane Cotin, Jeremie Dequidt and Mario Sanz Lopez are with INRIA and Lille University, France.

- Igor Peterlik is with Institut Hospitalier Universitaire (IHU) de Strasbourg, France and CERIT-SC, ICS, Masaryk University, Czech Republic.

- Erwan Kerrien and Marie-Odile Berger are with INRIA and Lorraine University, France.

E-mail: nazim.haouchine@inria.fr, stephane.cotin@inria.fr for MIS, many difficult problems have to be solved [1]. The abdominal cavity undergoing the laparoscopic surgery remains a very challenging environment for computer vision tasks. The surgical instruments interacting with the liver may cause large occlusions, the illumination variations caused by the endoscopic light and the liver bleeding or smokes due to electrocautery may disturb organ motion tracking and recovery of the 3D structures. Among the most difficult and still unsolved issues is the capacity to tackle, in real time, elastic deformations of the liver. This organ can undergo large deformations due to the interaction with surgical tools, respiratory motion and heart beating. Currently, most existing AR systems rely on rigid motion of the organs and only a limited number of papers address the problem of elastic organ deformation, considering only the homogeneous and linear elasticity.

The paper is organized as follows. Section 2 presents the previous works related to non-rigid registration and augmented reality for surgery. Sections 3, 4 and 5 explain our approach while section 6 details the experimental results obtained. Current results and limitations, as well as the applicability of the method are discussed in section 8 .

\section{Previous WORK}

In this section we present previous works related to augmented reality for Minimally Invasive Surgery. Since we propose to address the issue of elastic liver behaviour, we first present an overview of non-rigid registration methods in AR systems. Second, previous works on AR techniques for surgery guidance are presented. In the last part, we highlight our contribution.

\subsection{Capturing Non-rigid Surfaces}

Non-rigid surface augmentation has been a topic of interest of many researchers. The need to obtain realistic 
augmentation both in terms of geometric behaviour and in rendering has increased the number of papers in this area. Depending on the context, non-rigid registration and augmentation can be realized directly from 2D images [2] or may take advantage of a 3D model of the shape. When the texture information on the object is rich enough, many points can be matched between images, allowing a 2D deformation motion model to be computed. Bartoli et al. [3] used a direct method to register non-rigid pairs of images. In their approach, radial basis mapping is used as an equivalent to the optical flow constraint regularization approach [2]. In contrast to direct methods Pilet et al. [4] proposed a fast and robust tracking for handling large deformations. This approach uses a set of wide baseline feature matches and combines 2D deformable meshes with a robust estimation technique. Zhu et al. [5] proposed an approach to reduce the number of iterations of the previous method by using a progressive Finite Newton algorithm and an efficient factorization method to solve the optimization problem. In his approach Gay-Belille et al. [6] considered the occluded pixels as self-occlusion area that forces the wrap to shrink instead of outliers. Inspired by this self-occlusion shrinking method, Hilsmann et al. [7] proposed an approach exploiting an optical flow constraint regularization by a $2 \mathrm{D}$ deformable mesh. The advantage of such methods is that they can be applied with monocular images, but they require that rich information texture be available. Another restriction is that augmentation can only be realized onto the surface of the object. Thus, AR medical applications where virtual objects may be added in depth can not be handled by such techniques. To cope with this problem, a 3D deformable model has been considered. The deformation can be guided by monocular images [8] [9] or directly by $3 \mathrm{D}$ features available on the surface acquired with stereoscopic or depth camera as in [10].

One of the main difficulties of these approaches is to define an appropriate deformation model. Most of the time, it is computed from a representative sample of possible shapes using a dimensionality reduction process. However, sampling the space of valid shapes for quite deformable objects/organs is difficult. Another way to describe deformable shapes is to use mechanical-based models. In the past decade, many examples of mechanical-based tracking methods have emerged. Recent examples are for instance [11], where a combination of the finite element modelling with and the extended kalman filter shows the efficiency of the physics-based method. In [12], a linear finite element method is used to predict the deformation. The approach described in [13] relies on the minimization of a stretching energy subject to external image constraints. The problem is formalized by considering the surface compressibility (Poisson ratio) as the unique mechanical parameter assuming local linear elasticity.

Though theoretically well suited to describe the deformation of objects, the use of mechanical-based models is hampered by the difficulty to choose an appropriate mechanical model and the elastic parameters describing the considered deformations without over-parametrization. As shown in the next section, such models are promising in the medical field, especially because the volumetric segmentation is available as well as typical elastic parameters (Young's modulus) of numerous organs.

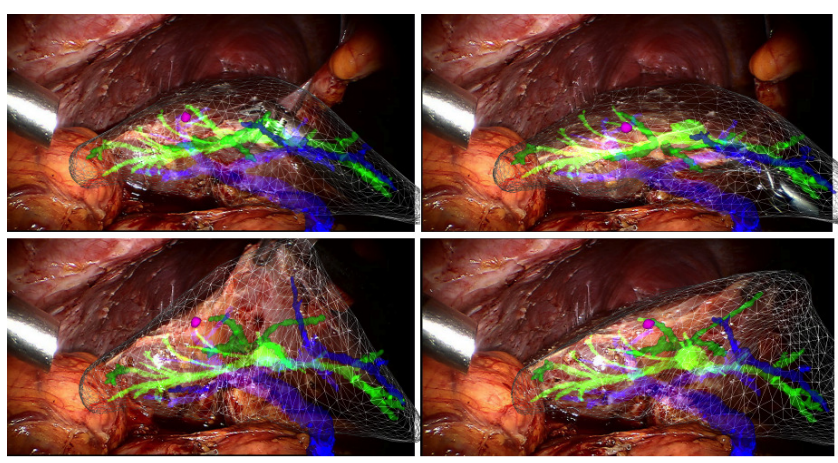

Fig. 1. A sequence of images showing the superimposition of the 3D real-time biomechanical model onto the human liver, undergoing deformation due to instrument interaction during minimally invasive hepatic surgery. The liver is represented in wire-frame, the tumor in purple, the hepatic vein is shown in blue and the portal vein in green.

\subsection{Augmented Reality for Surgery}

A number of research groups have investigated the use of AR during MIS, however, most contributions are dedicated to rigid organs or assume that elastic deformations between pre-operative and intra-operative data are negligible. In practice, large elastic deformations may occur due to breathing or due to the interaction of the organ with surgical tools. Past attempts to perform AR on deformable organs make use of markers or navigation aids placed close to the area of navigation targets [14] or require interaction to refine elastic registration between pre and intro-operative data [1]. Others, e.g. [15], build pre-operatively a dynamic 4D model of the heart which is registered to intra-operative data employing ECG. In [16], Su et al. propose a 3D-3D iterative closest point (ICP) registration with an image-based tracking to superimpose the 3D model onto laparoscopic images for partial kidney resection. Since the kidney does not actually undergo elastic deformations during surgery, the 3D-3D registration is performed in a rigid manner. These methods have shown the feasibility of automatic AR systems in surgery. Nevertheless, they usually require additional constraints during the intervention in the operating room or consider only simplified scenarios, for example cyclic or rigid motion of the organs.

Recently, significant advances have been realized towards automatic registration between pre- and intra-operative data in MIS. In the context of laparoscopy and employing the monocular images, Kim et al. [17] proposed a robust solution to track and augment a deformable surgical site using shape from shading and conformal mapping. However, their method only retrieves surface deformation and cannot 
ensure an accurate augmentation of inner structures. PuertoSouza et al. [18] proposed a matching algorithm called Hierarchical Multi-Affine capable of long-term tracking for augmentation during partial nephrectomy. This approach estimates a set of affine transformations from clusters of features in order to relocate occluded or missing features for a coherent spatio-temporal mesh registration.

When augmentation with in-depth structures is required, elastic registration of the surface of the organ is no more sufficient and volume tracking has to be considered.

Mechanical-based deformable models have proven to be relevant for volume deformation tracking since they allow to define elastic properties of the shape and thus to infer in-depth motion from boundary constraints. Nonetheless, simple models based on linear elasticity have been mostly considered [19], [20], [21]. In [22], a 4D scan of the heart is coupled with a biomechanical model. It is controlled by surface constraints created by features extracted from a stereovision stream and allows for a quite accurate overlay of internal structures. However, this approach is limited to cyclic movements where without large deformations due to the surgical manipulation.

In [23], a dynamic model based on the equation of dynamics for elastic materials is proposed: forces measured in the image drive the model towards the object's boundaries. The organ is supposed to be homogeneous and forces measured on MRI volumes, though noisy, are available everywhere in the structure. In [21], intraoperative registration between stereo endoscopic images and preoperative model of the liver based on biomechanical properties is proposed. The linear and Neo-hookean elastic models are considered and the accuracy of registration is assessed on a phantom. Results are slightly better with the second model, nevertheless it is still not suitable for a real time simulation.

A combination of physics-based model and image-based tracking was proposed in [24], [25] for vascular network augmentation during hepatic surgery. This approach employs a physical model as a regularization step to avoid false tracking measurement and to compute global volumetric deformations despite poor image-features detection. However, results are shown only for a very limited range of deformations, mainly when the deformation is perpendicular to the camera optical axis and only visual assessment is presented.

\subsection{Our Contribution}

This paper is an extension of our previous work published in [26] where we investigated the use of a realtime deformable liver model based on a biomechanical approach, and subject to constraints imposed by partial intra-abdominal image information. Here we present an extended experimental evaluation of this approach through two different, increasingly complex, scenarios, by using a high fidelity phantom liver data and intra-abdominal liver surgery data. These experiments permit to measure the internal positions of a tumor inside a tissue undergoing different types of deformations, with respect to a groundtruth. Moreover, we provide a comparison to the methods proposed previously. Since the stiffness and texture of the fabricated phantom and real liver differ, the in vivo tests on human data during hepatic surgery assess the robustness of the tracking algorithm when applied to a real scenario. Moreover, the initialisation step of the method, which still remains a challenge, is exposed in more details. The computational flow of the method is given in Fig. 2.

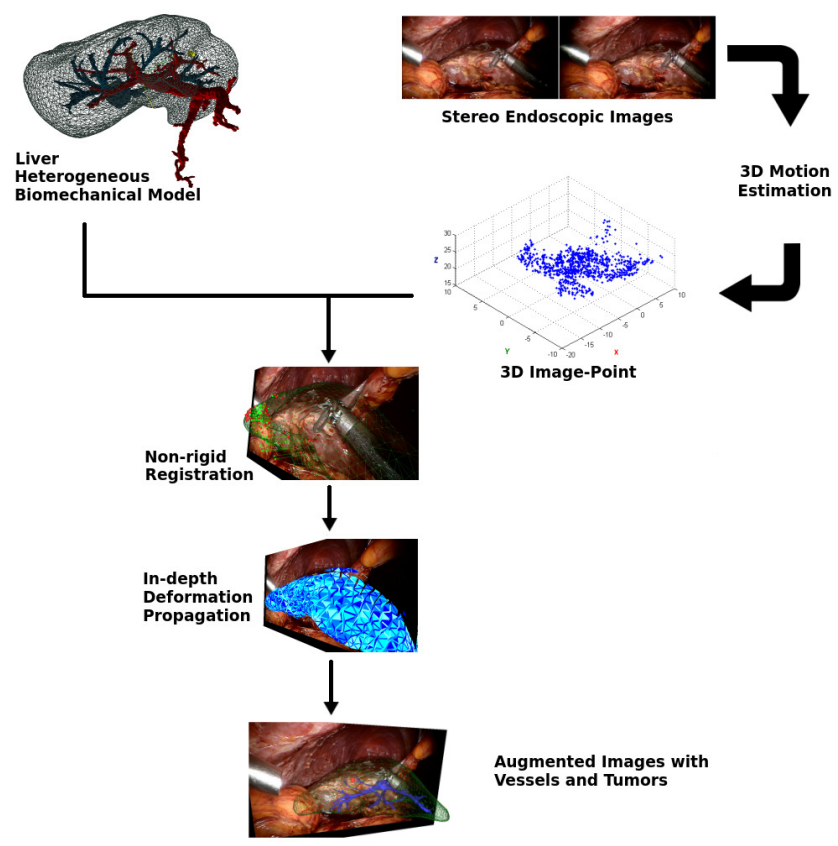

Fig. 2. The computational flow of the method: The biomechanical model guided by the 3D image-points recovered from the intra-abdominal image pair permits to propagate partial tissue deformations to vessels and tumors.

\section{3D Tissue Deformation Recovery AND TRACKING}

Capturing the 3D tissue deformation of an organ from intraabdominal images has been widely studied in the literature (see [27], [28] for a complete survey). In our work, a stereo endoscope is used to recover $3 \mathrm{D}$ information from the liver surface. In order to drive the simulation, our method requires a set of sparse 3D points. For that purpose, we use a feature-based tracking algorithm where salient landmarks are detected in each image pair using the Speeded-Up Robust Features (SURF) descriptor [29] and are tracked over time employing the Lucas-Kanade optical flow [30]. This combination has proven to be robust to track heart motion in laparoscopic images [31]. Other feature-matching frameworks can be used such as hierarchical frameworks presented in [18]. Feature correspondences between stereo images are obtained with a nearest neighbour criterion applied to the feature descriptors coupled with the epipolar constraint to filter out the outliers. After triangulation, we obtain a sparse set of $m$ 3D points, stored in a $3 \times m$ coordinate vector $\mathbf{y}$. Since our non-rigid registration method is a point-to-point registration, a sparse reconstruction is 


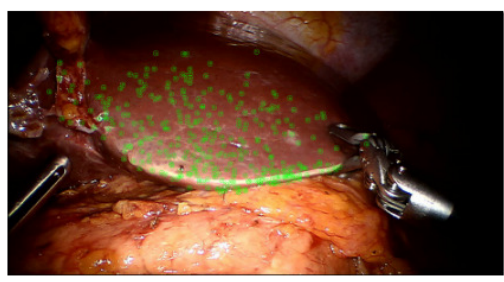

(a)

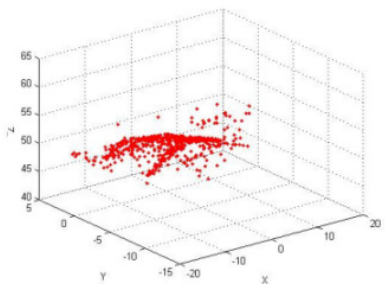

(b)

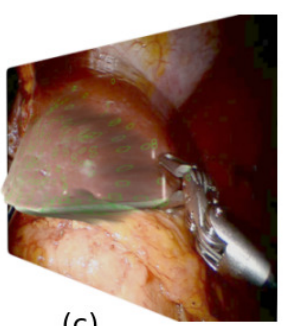

(c)

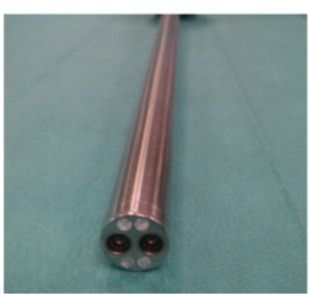

(d)

Fig. 3. 3D Estimation on a laparoscopic image of the abdominal cavity showing a part of the liver. (a) SURF features detection on image pairs acquired from the Da Vinci Robot. (b) The resulting sparse 3D point set $\mathbf{y}$ plotted and (c) mapped. (d) A stereo endoscope of the Da Vinci robot.

sufficient, however, the density of the point cloud can be increased in order to reduce outliers [32]. Examples of point correspondences and reconstructed 3D points from laparoscopic images are shown in Fig. 3.

We used a stereo endoscope from the Da Vinci robot illustrated in Fig. 3. The camera lens generate radial distortion and have relative small baselines (about $6 \mathrm{~mm}$ ). The camera is calibrated offline and the image distortions are rectified following Zhang [33]. We make the assumption that the stereo endoscope is fixed, which is the case when the surgeon manipulates the surgical instruments.

The SURF detector provides a measure of reliability for each extracted feature as the determinant of the Hessian matrix. We take advantage of this measure to associate each reconstructed 3D point with a quality $q$ defined as the average reliability of its two corresponding features in the left and right images. Quality values are normalized so that $q=1$ for the most reliable 3D point and $q=0$ for the least reliable $3 \mathrm{D}$ point in the point set $\mathbf{y}$. We denote $\mathbf{q}$ the $3 \times m$ vector formed by all values $q$, assuming the quality is isotropic at each point. Note that other measures of reliability could be considered, such as the Euclidean distances of the matched descriptors or the eigenvalues of the covariance matrix on the reconstructed points.

\section{Biomechanical Model}

In this section we provide a description of the biomechanical model used to compute the deformations of the liver. Before giving details of the tetrahedral model employed for parenchyma, we focus on the model of vascularization and mechanical coupling between these two components (see Figure 4). Since the final composite model is heterogeneous and anisotropic due to the vascular structures, we finally describe the solution process based on a direct solver, still allowing for real-time performance.

\subsection{Parenchyma Model}

Most biomechanical studies concerning the constitutive models of the liver parenchyma (see [34] or [35] for instance) report the non-linear and viscoelastic behavior of the organ tissue. Since we do not focus on the transient part of the deformation but rather the static equilibrium under some specific loading conditions, we do not take into account the viscous properties of the tissue. On the other hand, we aim at correct modeling of the large deformations, since during the surgical interventions, an important displacements of tissue (e.g. the liver lobes) occur due to the action of the surgical tools.

For this reason we have opted for the finite element method based on a co-rotational formulation (introduced by Felippa in [36]) allowing for large displacements while relying on a linear expression of the stress-strain relationship. The co-rotational approach is based on the decomposition of the actual element configuration into rotational and deformational components, both being quantified w. r. t. the initial position. More precisely, the actual position of the element nodes determines the base of the element (given by three chosen adjacent edges), which is both rotated and deformed w.r.t. the initial base of the same element. In order to extract the rotational component (denoted as $\mathbf{R}_{e}$ ), a matrix decomposition such as polar, SVD or QR is needed; in our method we employ the technique described in [37]. The matrix $\mathbf{R}_{\mathbf{e}}$ is used to update the local stiffness matrix $\mathbf{K}_{\mathbf{e}}$ of the element. Therefore, via this element-wise rotations, the actual global stiffness matrix $\mathbf{K}$ depends in each step on the actual deformation $\mathbf{u}$ and the equation relating the external forces to the displacements can be written as

$$
\mathbf{f}=\mathbf{K}(\mathbf{u}) \mathbf{u} \text { with } \mathbf{u}=\mathbf{x}-\mathbf{x}^{0}
$$

where $\mathbf{x}_{0}$ and $\mathbf{x}$ represent nodal positions in rest and actual positions, respectively, and $\mathbf{f}$ are the external forces.

Assuming that linear tetrahedral P1 elements are employed in the finite element formulation of the parenchyma model and the mesh is composed of $N_{\mathbb{P}}$ nodes, the resulting system has $3 N_{\mathbb{P}}$ degrees of freedom, i. e. $\mathbf{u}_{\mathbb{P}}, \mathbb{P}$ are vectors of size $3 N_{\mathbb{P}}$ whereas $\mathbf{K}_{\mathbb{P}}$ is a $3 N_{\mathbb{P}} \times 3 N_{\mathbb{P}}$ matrix, where the subscript $\mathbb{P}$ denotes the parenchyma.

It should be noted that our method is not limited to the co-rotational model, which can be replaced by hyperelastic formulation such as the technique based on multiplicative Jacobian energy decomposition presented in [38].

\subsection{Vessel Model}

The vascular system is regarded as the main source of heterogeneity which has a global influence on the mechanical response of the vascularized tissue due to important stiffness of the vessel wall. The model employed here is 
based on work presented in [39]. Besides describing the model in the actual scenario, additional details concerning the assembling of the composite system are given in the following text. It should be emphasized that a potential viscoelastic response due to fluids (i.e. blood) circulating in the vessels is not taken into account as only a quasi-static scenario is assumed.

From the mechanical point of view, the vascular system is modeled with serially linked beam elements in a similar way as proposed by Duriez et al. [40] for simulating catheters and guide wires. This model shares some similarities with the co-rotational model described above, and in particular allows for geometrically non-linear deformations. At the same time it accounts for rotational degrees of freedom, i. e. besides linear positions and forces, orientation and torques are included in the mechanical formulation.

We introduce some modifications to the model to take into account the nature of vessels, in particular through specific cross section profiles and moments of inertia (see [41] for details). The static formulation for the deformation of a beam is described by a system similar to Eq. 1 with constituents $\mathbf{u}_{\mathbb{V}}, \mathbf{f}_{\mathbb{V}}$ and $\mathbf{K}_{\mathbb{V}}$. However, as each node is equipped with 6 degrees of freedom due to the rotational components, the size of vectors $\mathbf{u}_{\mathbb{V}}$ and $\mathbf{f}_{\mathbb{V}}$ is $6 N_{\mathbb{V}}$ and $\mathbf{K}_{\mathbb{V}}$ is a $6 N_{\mathbb{V}} \times 6 N_{\mathbb{V}}$ matrix where $N_{\mathbb{V}}$ is the size of beam mesh representing the geometry of vessels.

\subsection{Coupling between Vessels and Parenchyma}

In order to build the composite model of vascularized tissue, we adopt the method proposed in [39]. At the beginning of the time step, the forces applied in the beam points are propagated to the vertices of tetrahedra. Then, stiffness matrices for both beam and tetrehedral models are assembled and combined together as described below. The composite system is solved resulting in displacements of the tetrahedral vertices. Finally, the positions of beam nodes are updated based on actual positions of tetrahedra. For the sake of simplicity, we first describe the positional mapping between the nodes, then the propagation of forces and finally we show how the stiffness matrices are combined together to get the global stiffness matrix of the composite system.

Let us first focus on positional mapping of an arbitrary beam point of the vessel network. We recall that the point is defined by its position $\mathbf{p}$ and orientation $\mathbf{O}$ in space. Before the simulation starts, the point is associated with a tetrahedron $T$ which contains the point. Two quantities are precomputed for each point: the barycentric coordinates of p w.r.t. the tetrahedron $T$ (denoted as $\beta_{T}$ ) and orientation $\mathbf{O}_{T}$ of the point formulated relatively with respect to the tetrahedron $T$, computed as $\mathbf{O}_{T}=\mathbf{B}_{T}^{-1} \mathbf{O}$ where $\mathbf{B}_{T}$ is an orthogonal base given by the edges connecting the nodes of the tetrahedron $T$ in the rest position. It should be noted that the same matrix is required by the corotational model. Since it is supposed that no relative motion between the parenchyma and vessels occurs, both $\beta_{T}$ and $\mathbf{O}_{T}$ remain constant during the simulation and in each step they are used to calculate the updated position $\mathbf{p}$ and orientation $\mathbf{O}$ of the beam point in space. As for the position, the calculation using the barycentric coordinates is straightforward. The updated orientation $\mathbf{O}^{\prime}$ is computed as $\mathbf{O}^{\prime}=\mathbf{B}_{T}^{\prime} \mathbf{O}_{T}$ where $\mathbf{B}_{T}^{\prime}$ is the base given by update positions of the tetrahedron $T$. The calculation of the updated base $\mathbf{B}_{T}^{\prime}$ requires the orthogonalization process, however, this computation is already needed by the co-rotation model, so the mapping does not introduce an additional significant computational load.

At the same time step, the response forces applied in the beam node are mapped onto the vertices of the associated tetrahedron $T$. For the beam point, there are linear forces f as well as torques $\tau$, whereas for the tetrahedron, only linear forces $\mathbf{F}$ are modeled in the element vertices. The linear response force $\mathbf{f}$ is mapped from the beam point to its corresponding tetrahedron $T$ using the barycentric coordinates $\beta_{T}$ introduced above. The torques $\tau$ are transformed to the linear forces acting in the tetrahedron nodes using the equation $\tau=\mathbf{r} \times \mathbf{F}$ where $\mathbf{r}$ is vector connecting the beam and tetrahedron nodes. Putting it together, the force contribution of the beam point is added to the forces applied in the vertices of associated tetrahedron $T$ as $\mathbf{F}_{i}=\mathbf{F}_{i}+\beta_{i} \mathbf{f}-\left(\mathbf{r}_{i} \times \tau\right)$ where $i$ runs over the vertices of $T$.

As introduced above, the vascularized tissue is a composite deformable object, where the total stiffness matrix $\mathbf{K}$ is composed of contributions provided by stiffness of parenchyma $\mathbf{K}_{\mathbb{P}}$ and stiffness of vessels walls $\mathbf{K}_{\mathbb{V}}$. Let us suppose that the mapping between the beam and tetrahedra nodes can be be expressed in a matrix form as $\mathbf{f}_{\mathbb{P}}=\mathbf{J}^{T} \mathbf{f}_{\mathbb{V}}$ where $\mathbf{J}$ is a $3 N_{\mathbb{P}} \times 6 N_{\mathbb{V}}$ Jacobian matrix of the mapping between the nodes of parenchyma and vessels [42]. The global stiffness $\mathbf{K}$ matrix is then computed as $\mathbf{K}=\mathbf{K}_{\mathbb{P}}+\mathbf{J}^{\top} \mathbf{K}_{\mathbb{V}} \mathbf{J}$.

Concerning the tumor, since this work focus only on small tumors, we assume that its influence on the overall mechanical behaviour is negligible and therefore the coupling with the parenchyma is only geometric. However, the tumor can easily be modelled as a real mechanical object with different properties from those of the parenchyma, which will not affect the performance of our method.

\subsection{Numerical Solution}

In this paper, a quasi-static scenario is considered, i. e. the actual shape of deformable object under applied forces is computed using the finite element formulation without dealing with the dynamic properties of the tissue. Therefore, in each step of the simulation, a linear system given by Eq. 1 is resolved. A wide range of direct and iterative solvers have been proposed in the past to solve such system of equations emerging in the physics-based modeling of deformable bodies. In the case of homogeneous systems where the finite element formulation results in well-conditioned matrices, iterative solvers such as conjugate gradients have proven to be efficient techniques converging rapidly to the optimal solution. However, in our case, the final matrix $\mathbf{K}$ gathers mechanical contributions of both the parenchyma and vessel walls. As the experiments reports a significant difference 


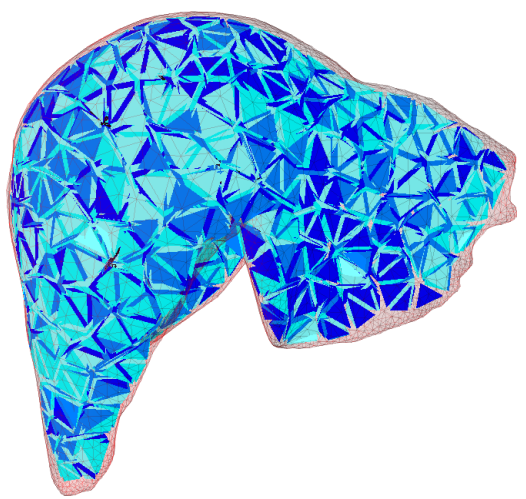

(a)

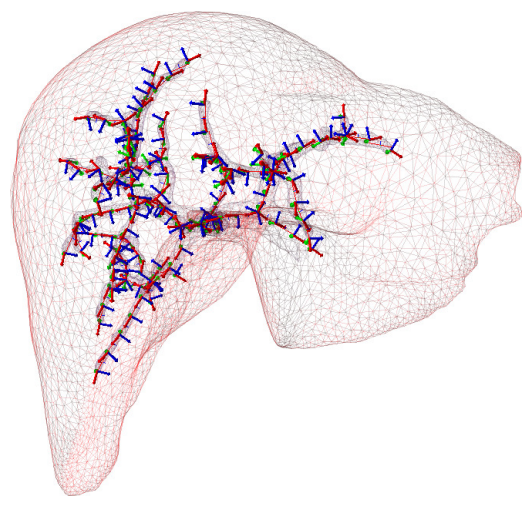

(b)

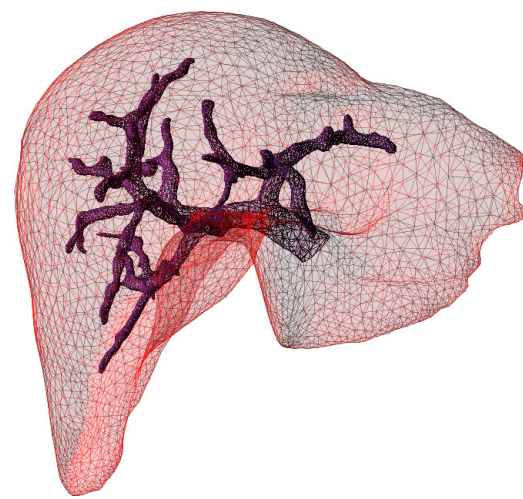

(c)

Fig. 4. 3D heterogeneous biomechanical model of the liver: (a) The volumetric mesh composed of tetrahedra. (b) The beams generated along the vessels described in Section 4. (c) The heterogeneous liver model including the vascular network shown in wire-frame.

in stiffness of these two components (e.g. see [43]), the composite system results in poorly conditioned matrix. In this case the convergence of iterative solvers becomes an important issue.

For this reason, we rely on direct LDL solver which requires an explicit factorization of the system matrix. Although the solver imposes more strict limitations on the size of the system being resolved, it still provides a stable real-time solution applicable to the problems considered in the scope of this paper.

\section{NON-RIGID REgISTRATION}

\subsection{System initialization}

Special care must be taken during the initialization phase since it can significantly impact the estimated position of the tumor. As in related works [16], [18] the initialisation is done manually through a Graphical User Interface (GUI).

First, in order to ensure visual consistency, the intrinsic parameters of the endoscopic camera are loaded on the GUI virtual camera. Dense 3D reconstruction is built on the first pair of laparoscopic images (the first pair is chosen so that a large part of the liver is visible). This dense reconstruction permits to obtain a set of partial patches that represent the visible liver surface. The liver mesh is then aligned manually to the reconstructed surface, based on salient geometrical landmarks such as liver contours or surrounding ligaments. This alignment consists in a rigid transformation (rotation and translation) and is therefore insufficient to initialise the registration correctly. Indeed, due to the gas insufflation, the pre-operative data (liver mesh from the segmented CT scans) geometrically differs form the intra-operative data (reconstructed patch) [44]. To solve this issue, we constraint the biomechanical model (including the vessels and the tumour) with external forces so that it fit better the recovered visible liver surface.

The temporal non-rigid registration is a point-to-point registration (cf. Fig. 5), therefore, the set of threedimensional points reconstructed using this initial stereoscopic pair of images is projected onto the liver model surface. This is done using a ray casting method, followed by a computation of barycentric coordinates describing the position of these points with respect to adjacent degrees of freedom of the biomechanical model. Only features that intersect the liver surface after ray-casting are kept, the features that do not belong to the liver are filtered out from laparoscopic images. Finally, the physical boundary conditions of the biomechanical model are set by fixing its correct degrees of freedom.

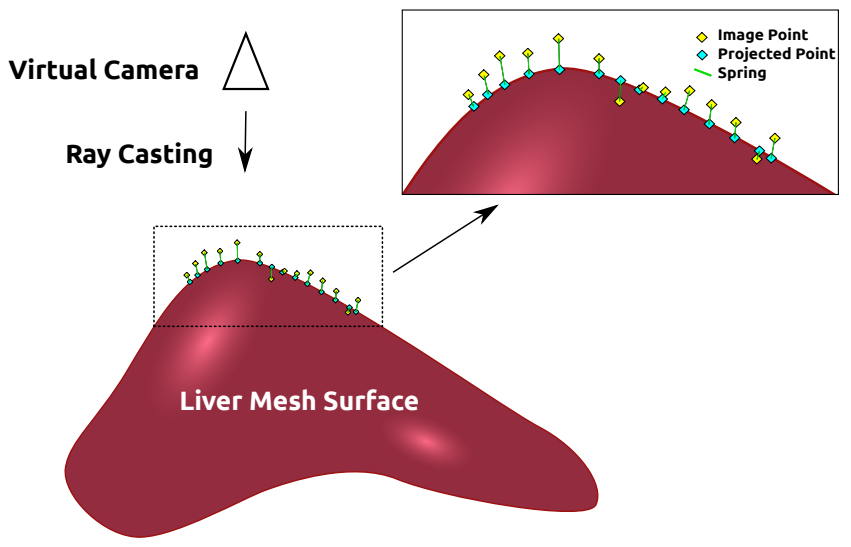

Fig. 5. Point-to-point registration: The initial set of tracked features point $\mathbf{y}$ is projected on the surface using ray-casting to pair the set $\mathbf{y}$ with the degrees of freedom of the mechanical model $\mathbf{x}$ at $t=0$.

\subsection{Stretching Energy Minimization}

The non-rigid registration can be seen as a stretching energy minimization between the three dimensional features recovered from laparoscopic images that represent the tracking energy $E_{t}$ and the biomechanical model derived from the preoperative CT data. We consider an energy term that accounts for the internal energy of the biomechanical model $E_{i}$ and the tracking energy $E_{t}$. Deriving this energy shows that an extreme (minimum) is reached when the internal 
forces equal the tracking forces. Thus, the internal forces are expressed as:

$$
\mathbf{f}_{\mathbf{i}}(\mathbf{x})=\mathbf{R}_{\mathbf{e}} \mathbf{K} \cdot\left(\mathbf{R}_{\mathbf{e}}^{\top} \mathbf{x}-\mathbf{x}^{\mathbf{0}}\right)
$$

where $\mathbf{K}$ represents the stiffness matrix, $\mathbf{R}_{\mathbf{e}}$ the co-rotational matrix and $\mathbf{x}, \mathbf{x}^{\mathbf{0}}$ are vectors of size $3 n$ representing the position of the $n$ degrees of freedom of the mechanical model, respectively at any time $t$ and time $t=0$.

The tracked 3D features $\mathbf{y}$ represent how visible parts of the liver are moving. Due to the noise or other reconstruction inaccuracies, point locations in $\mathbf{y}$ are often regularized by generating a smooth and dense surface model that is later used as boundary conditions for the biomechanical model [21]. Instead, we propose here to incorporate such inaccuracies directly in the mechanical solver using quality values measured at each $3 \mathrm{D}$ point.

External forces are defined by pairing the $m$ 3D points $\mathbf{y}$ to the $n$ degrees of freedom $\mathbf{x}$ of the bio-mechanical model. As explained in 5.1, the tracked points $\mathbf{y}^{\mathbf{0}}$ at initialization $(t=0)$ are expressed in barycentric coordinates of the adjacent degrees of freedom leading to the linear relation:

$$
\mathbf{y}^{\mathbf{0}}=\mathbf{L} \cdot \mathbf{x}^{\mathbf{0}}
$$

where $\mathbf{L}$ is a rectangular $3 m \times 3 n$ matrix. We assume this linear relation remains valid during the deformation. At any later time $t$, the stretching forces induced by the tracking are defined as

$$
\mathbf{f}_{\mathbf{t}}(\mathbf{y})=k \cdot \mathbf{q} \cdot\left(\mathbf{y}-\mathbf{y}^{\mathbf{0}}\right)
$$

where $k$ can be seen as a stiffness. Finally, the stretching forces can be expressed with respect to the degrees of freedom as

$$
\mathbf{f}_{\mathbf{t}}(\mathbf{x})=\mathbf{L}^{\top} \cdot \mathbf{f}_{\mathbf{t}}(\mathbf{y})=\mathbf{f}_{\mathbf{t}}(\mathbf{x})=\mathbf{L}^{\top} \cdot k \cdot \mathbf{q} \cdot \mathbf{L} \cdot\left(\mathbf{x}-\mathbf{x}^{\mathbf{0}}\right)
$$

Parameter $k$ is set to be one or two order of magnitude higher than the Young's modulus in order to impose the boundary conditions directly and to insure that the simulation remains stable even at large integration steps.

\section{Experimentation With Liver Phan- TOM DATA}

In laparoscopic surgery, a reproducible validation remains a challenging task. In our case, the problem is more complex since neither qualitative results nor visual assessment can be used for validation easily, since the internal structures are not visible in the laparoscopic images. We believe that performing a CT scan of a phantom liver during the deformation is an ideal way of defining a ground truth for the location of an internal structure (e.g. a tumor). However, surgical instruments as well as the laparoscopic camera produce significant artifacts during a $\mathrm{CT}$ in vivo acquisition. Further, the boundary conditions of the liver surface, which influence the motion and deformation of the organ significantly, are difficult to identify reliably. Therefore we designed a validation protocol in order to quantify the error of the prediction computed by the method proposed in this paper. In the following, we first describe the bench used to validate our simulation by providing details about the phantom construction and desired properties. Second, we explain how the ground truth is determined and finally, we present a detailed comparison and evaluation of the results.

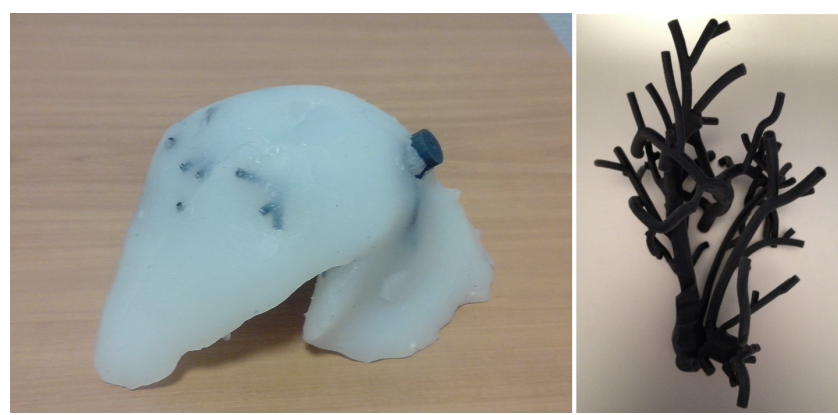

Fig. 6. The heterogeneous liver phantom: the vascular network (right) is considered as the main source of heterogeneity of the silicon-made phantom (left).

\subsection{Bench Description}

\subsubsection{Required Properties}

Quantitative validation strongly depends on the targeted application. The ex-vivo setup was designed to mimic as closely as possible a typical difficulty encountered during liver tumor resection in laparoscopy: The liver is firmly grasped and the tumor locations must be predicted despite important deformations. The first two features concern the liver phantom that should be based on a patient-specific data, encompass the vasculature as well as tumors, and display known mechanical properties similar to that of a human liver. The ground-truth tumor locations are tracked with successive CT scans of the liver acquired while a progressive force is applied. The phantom and the global setup should be compatible with CT imaging. The applied force should be controlled to be reproduced in the simulation. Moreover, the reproducibility of the deformation is important also because of the fact that recording the laparoscopic video and $\mathrm{CT}$ acquisition cannot be done simultaneously. Therefore, each is performed separately, however, both modalities are applied to the object undergoing exactly the same deformation. Finally, the grasping device should not occult video images and deformations should be varied, which requires the ability to modulate force direction and amplitude.

\subsubsection{Model Fabrication}

To the best of our knowledge, the known polymers and similar materials are not capable of reproducing the behavior of liver tissue with high fidelity. However, extremely soft silicone rubber seems to be a good choice being close enough to a real liver properties, having similar density and good maximal elongation before a failure occurs [45]. Using a surface representation of a real patient data (obtained using semi-automatic methods provided by ITK-Snap ${ }^{1}$, we

1. http://www.itksnap.org 
employed 3D printing to reproduce the exact geometry of the organ and the vascular network.

Given the stiffness of the vessel wall reported for example in [43], the network was produced directly by 3D printing using the softest available polymer (DM9895) having the Shore hardness $A=90$. The fabrication of the parenchyma was performed using a mold made in a Siligum to reproduce the liver shape. The mold was further filled with an extremely soft silicone rubber mix using Ecoflex $\AA$ 00-20 and additive Slacker $\AA$ in ratio $1: 1 / 8$ by volume. During the filling procedure, the printed vascular networks together with artificial inclusions (representing randomly placed tumors) where inserted inside the mold. The phantom is shown in Fig. 6.

The procedure results in a very soft and elastic material representing the parenchyma with a harder vascular network placed inside. Each component is easily detectable on a CT scan in order to perform an automatic segmentation. The Tab. 1 shows the values of the most significant parameters for each component. Finally, the phantom is manually textured with salient marks in order to ensure a robust tracking.

\begin{tabular}{llll} 
& Vessels & Parenchyma & Tumor \\
\hline Mass (gr) & 30 & 675 & 0.2 \\
\hline Volume (cc) & 32.9 & 690.2 & 6.3 \\
\hline Largest dimension (mm) & 148 & 179 & 3 \\
\hline Young's Modulus (MPa) & 9.2 & 0.05 & - \\
\hline Poisson's ratio & 0.45 & 0.40 & - \\
\hline
\end{tabular}

TABLE 1

Characteristics of the liver phantom.

\subsubsection{Bench Design and Construction}

Considering the specification and constraints formulated above, we apply a deformation so that the errors introduced due the friction between different parts of the setup be minimized. To induce the deformation, we use a servomotor BMS-2514 capable of a torque up to $23.2 \mathrm{~kg} . \mathrm{cm}$, attached to a pulley having $71 \mathrm{~mm}$ in diameter. Three different threads attached to the same region of the liver surface using graspers are used to pull in three different directions aligned to $x, y$ and $z$ axes as seen in the camera view. The effective force up to $64 \mathrm{~N}$ can be applied on the liver surface via this mechanism depicted in Fig. 7.

Three different deformations induced by the threads are depicted in Fig. 7: in Deformation 1, a lateral pulling is applied, in Deformation 2, the surface is being pulled upwards and in Deformation 3, pulling towards the camera is performed.

To ensure the compatibility with X-ray based imaging, every element close to the liver is made of low density substances. More precisely, each grasper as well as the two closest pulleys have been specially designed and 3Dprinted in PLA plastic, chosen for its high rigidity compared to other thermoplastics, providing reliability and stability

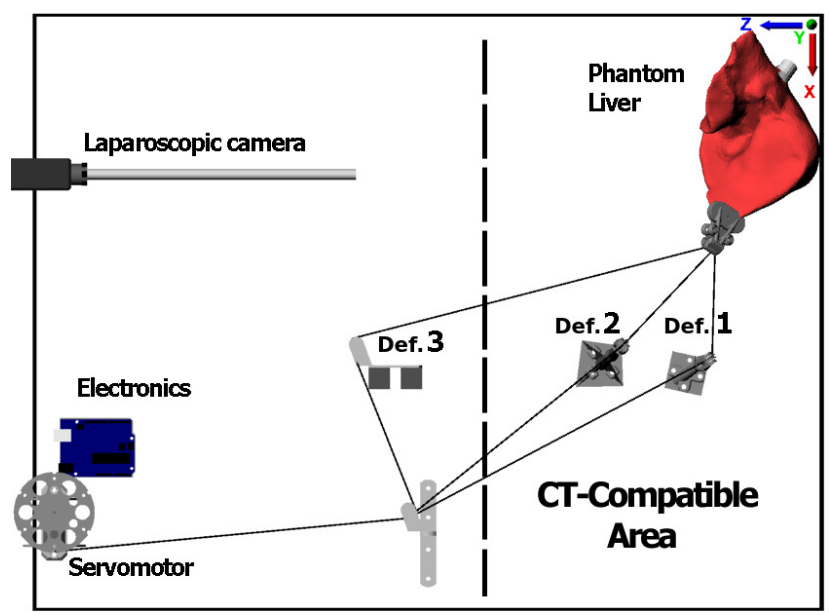

(a)

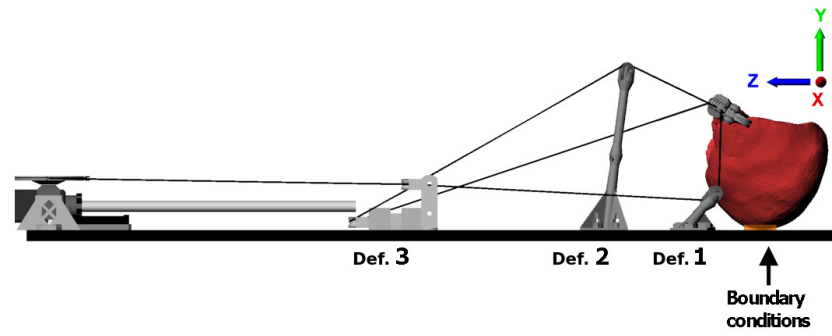

(b)

Fig. 7. Validation bench with top (a) and side (b) views. The bench is CT-compatible and allows for easy reproduction of the experiments. The three deformations permit to quantify the errors depending on the direction and elongation and at the same time, they can be simulated straightforwardly via Dirichlet boundary conditions.

during the output deformation. Polyamide bolts have been used to fix every element to the base platform.

Arduino-based micro-controller is used to control the servomotor which is driven according to the commands received from a wired electromagnetically isolated control pad. The control is also visualized with a LED which is used for synchronization. The liver is fixed to the bench using a glue to ensure fixed boundary conditions.

\begin{tabular}{llll} 
& Def. 1 & Def. 2 & Def. 3 \\
\hline Elongation $(\mathbf{m m})$ & \multicolumn{3}{c}{ Force $(\mathbf{N})$} \\
\hline 18.8 & 3.13 & 3.13 & 0.93 \\
\hline 37.7 & 6.47 & 6.72 & 2.06 \\
\hline 56.5 & 8.87 & 9.27 & 4.12 \\
\hline 75.4 & 12.65 & 12.94 & - \\
\hline \multicolumn{4}{c}{}
\end{tabular}

Elongation and forces applied for each deformation.

\subsection{Ground Truth Acquisition}

The ground truth acquisition involves two procedures described in Fig. 8): 


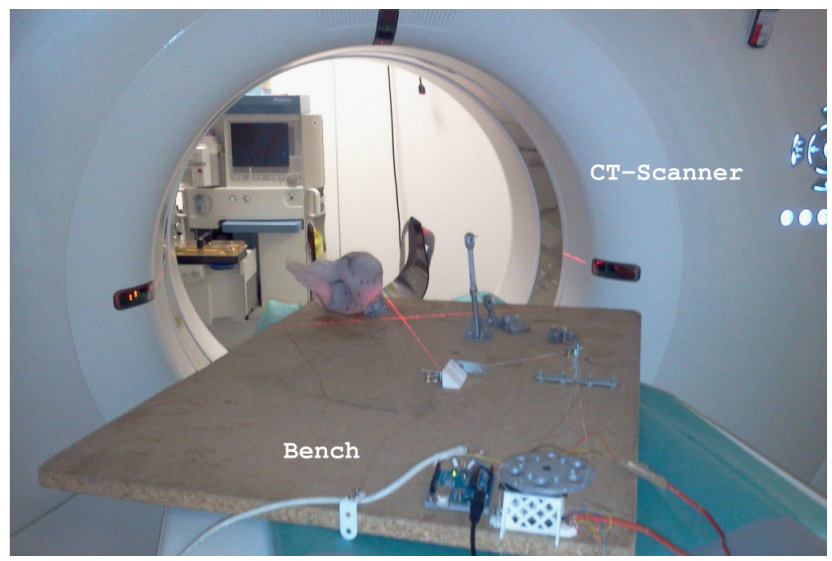

(a)

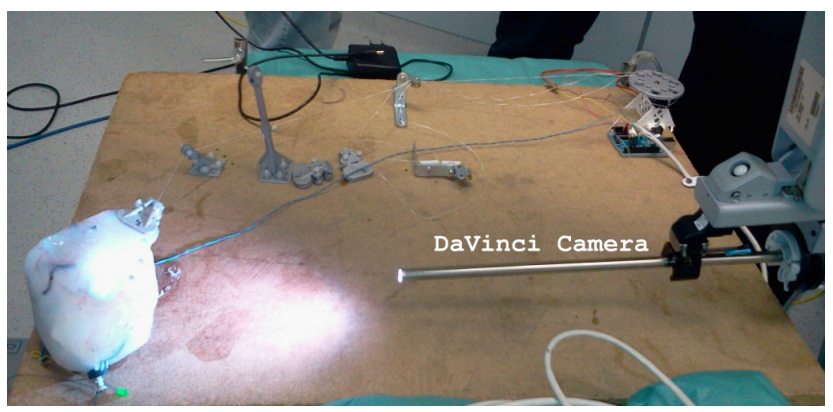

(b)

Fig. 8. Experimental protocol for the quantitative validation: (a) The CT scanner with the bench. The wire hooked to the lobe is pulled by the servomotor and maintained in position while performing a new CT scan to provide a ground truth deformation. (b) An overview of the stereoscopic camera acquiring video stream of the deformation.

- CT scanning: we perform a CT scan of the phantom, being first at undeformed configuration, and then at different deformed configurations (cf. Tab. 2). The scans are acquired with the Siemens Somatom CT Scanner producing high quality volume images.

- Video streaming: we record the deformation induced by the pulling threads using a stereo endoscopic camera. The video stream is recorded at 29.97 FPS producing $960 \times 540$ images acquired from the DaVinci Robot from Intuitive Surgical $\AA$.

The two procedures are repeated for each deformation, so a sufficiently large data set is obtained giving the exact locations of the tumors (center of gravity) and the corresponding stereo endoscopic views. A result of the threedimensional reconstruction from CT scans is illustrated in Fig. 13.

\subsection{Model Comparison and Quantitative Results}

In order to justify the employment of a heterogeneous biomechanical model, a comparison with a homogeneous model is provided for three different tumor locations: near the point of traction (Tumor 1) and surrounded by vessels
(Tumor 2 and Tumor 3) (illustrated in green in Fig. 13). Additionally, a comparison to a mass-spring model is given. It should be emphasized that the comparison is done with the same data and parameters and using the same initial registration.

We calculate the Euclidean distance between the center of the mass of the scanned tumor and the center of the mass of the tracked tumor. The results are presented in Fig. 9,10,11,12.

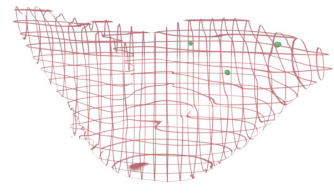

(a)

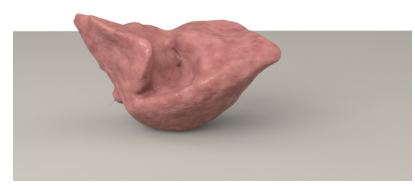

(c)

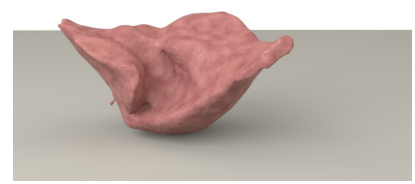

(e)

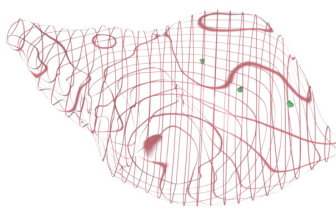

(b)

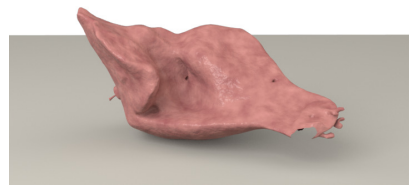

(d)

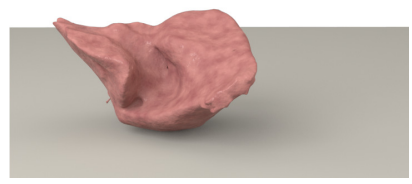

(f)
Fig. 13. CT-scans: the liver with the initial locations of tumors (green spheres) in front (a) and top (b) views and the $3 D$ reconstructed phantom from CT images at rest (c) and deformed in Deformation 1 (d), Deformation 2 (e) and Deformation 3 (f).

\subsubsection{Deformation 1}

In this deformation the lobe is mainly pulled along $x$ and $z$ axes. The heterogeneous model gives the lowest errors when compared to the homogeneous and the mass-spring models. The largest difference between the models can be noticed in the case of Tumor 2 and Tumor 3 that are surrounded by vessels. Also, for these two tumors, the error remains under $6 \mathrm{~mm}$ despite the increase in the elongation. The average error for Tumor 1 is $7.40 \mathrm{~mm}$, Tumor 2 $2.79 \mathrm{~mm}$ and Tumor $35.46 \mathrm{~mm}$, being significantly smaller that corresponding errors obtained with homogeneous and mass-spring models (cf. Fig. 12).

\subsubsection{Deformation 2}

In this case the liver lobe is subjected to an elongation along $y$ and $z$ axes. Similarly as in the previous Deformation 1 , the heterogeneous model gives the lowest errors when compared to the homogeneous and the mass-spring models. However, in Tumor 1, the error in heterogeneous model remains important $(9.26 \mathrm{~mm})$ and is similar to the results obtained using the other two models. Moreover, it increases 


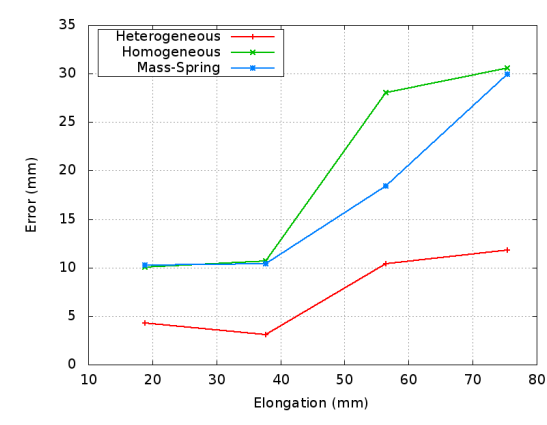

(a)

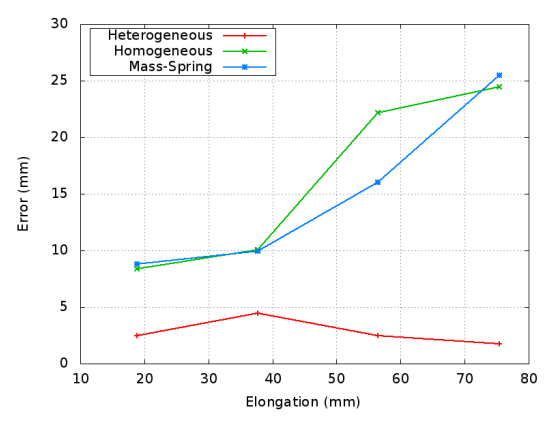

(b)

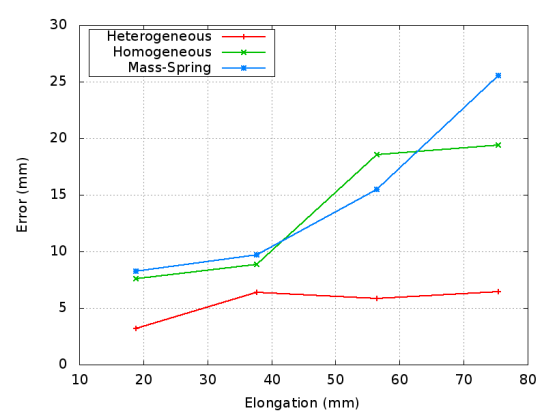

(c)

Fig. 9. A comparison of homogeneous FEM model, heterogeneous FEM model and mass-spring model for the Deformation 1 with: Tumor 1 (a), Tumor 2 (b), Tumor 3 (c).

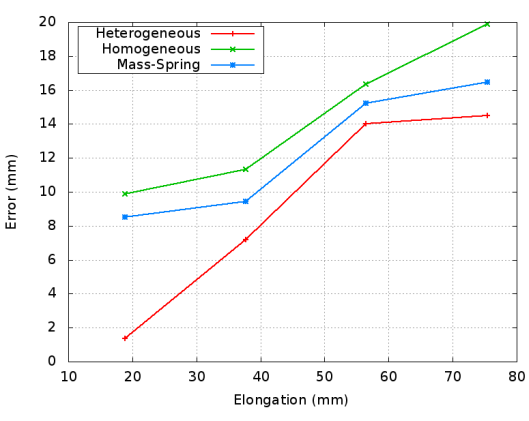

(a)

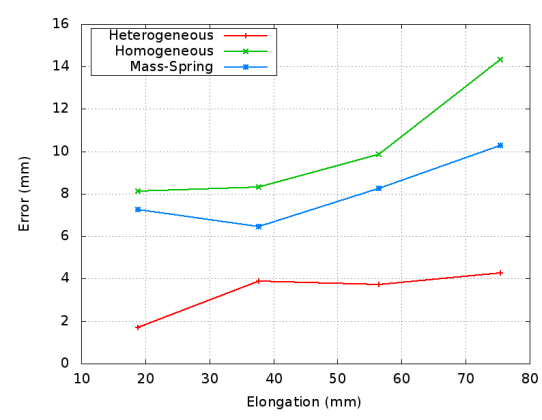

(b)

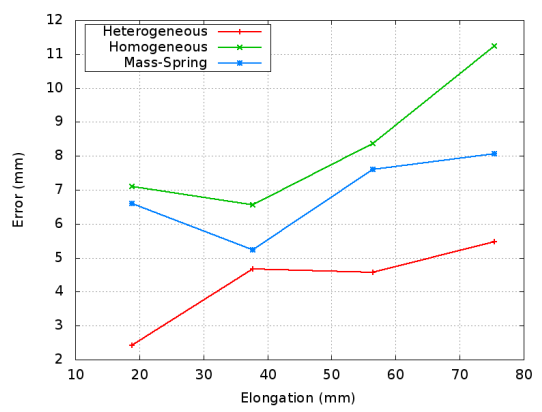

(c)

Fig. 10. A comparison of homogeneous FEM model, heterogeneous FEM model and mass-spring model for the Deformation 2 with: Tumor 1 (a), Tumor 2 (b), Tumor 3 (c).

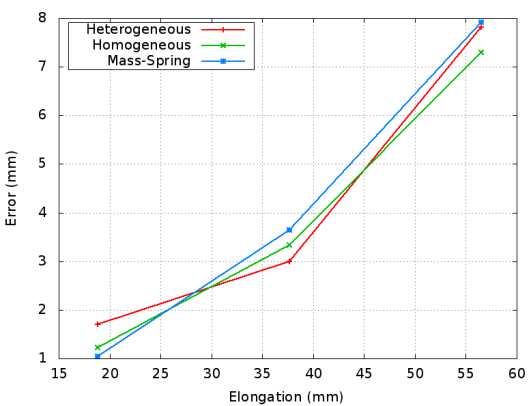

(a)

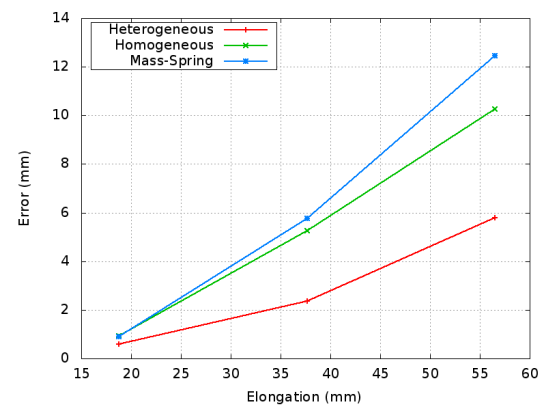

(b)

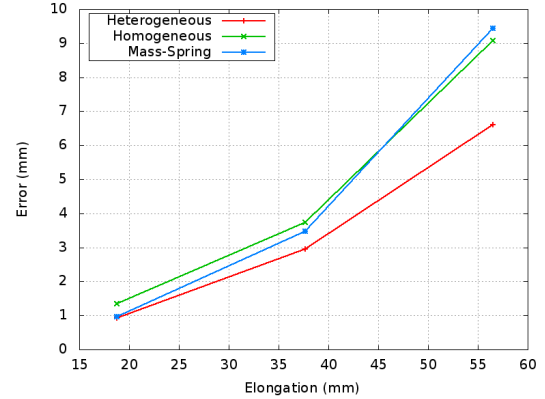

(c)

Fig. 11. A comparison of homogeneous FEM model, heterogeneous FEM model and mass-spring model for the Deformation 3 with: Tumor 1 (a), Tumor 2 (b), Tumor 3 (c).

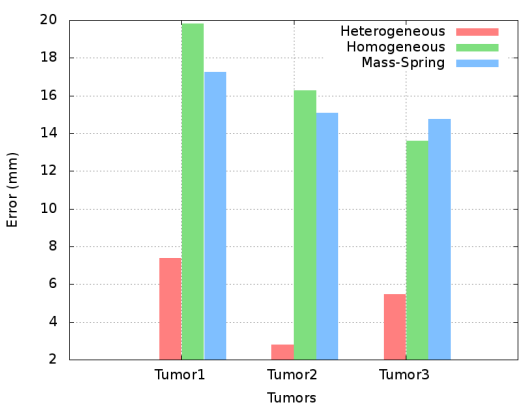

(a)

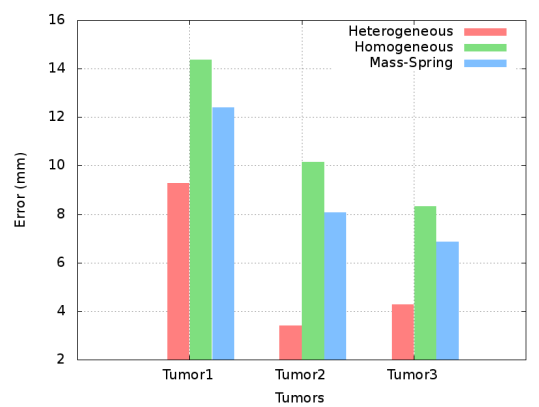

(b)

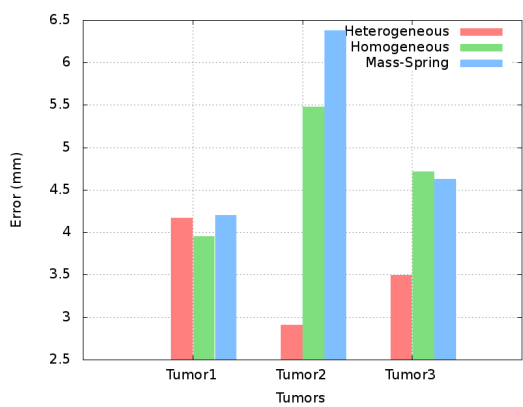

(c)

Fig. 12. Average error for Deformation 1 (a), Deformation 2 (b) and Deformation 3 (c). 
with the elongation. It should be noted that the $z$ axis shows the highest uncertainty in the $3 \mathrm{D}$ reconstruction which might partially explain the result, together with the magnitude of the external applied force. The average error for Tumor 2 is $3.39 \mathrm{~mm}$ and Tumor $34.28 \mathrm{~mm}$.

\subsubsection{Deformation 3}

The purpose of the Deformation 3 is to pull one lobe of the liver in the direction of the camera. Even if the average errors for the three tumors are low, $(4.17 \mathrm{~mm}, 2.91 \mathrm{~mm}$ and $3.49 \mathrm{~mm}$ ), we notice a similarity between the three models which can be explained by the fact that the deformation undergone by the liver is close to a rigid transformation (mainly a rotation). The vascular network effect on the behaviour is therefore reduced.

The overall results show that the use of a heterogeneous model reduces the distance between the scanned tumor and the simulated one considerably. These errors depend on the location of the tumor and the type of deformation. We can also notice that the homogeneous model may display a similar behaviour to that of mass-spring model under certain circumstances (such as quasi-rigid deformation).

We believe that the reported results show a significant improvement over the current laparoscopic procedures since a safety margin between $10 \mathrm{~mm}$ and $25 \mathrm{~mm}$ around the possible location of the tumor (estimated from the preoperative CT scan) is usually considered [46].

\section{Results on in vivo Human Liver}

When dealing with an actual patient, quantitative validation implies major technical challenges as well as ethical issues. Our approach was conducted on a laparoscopic sequence taken during a real examination. Our aim was to demonstrate the accuracy of the tracking in a real environment (specular lights, beating heart, respiratory motion, instrument occlusions, and difference between porcine and human liver textures) and the performance of our non-rigid registration. In addition, since the texture of the phantom may bias the tracking robustness, in vivo experimentations are needed.

We employ the values of the elastic parameters according to [47], [43], [39]: Young's modulus of parenchyma $E_{\mathbb{P}}=27 \mathrm{kPa}$ and Poisson's ratio $v_{\mathbb{P}}=0.45$, Young's modulus of vessel wall: $E_{\mathbb{V}}=0.62 \mathrm{MPa}$ and Poisson's ratio $\nu_{\mathbb{V}}=0.45$, thickness of the vessel wall: $t_{\mathbb{V}}=200 \mu \mathrm{m}$. The model was composed of 3391 linear P1 tetrahedral elements (parenchyma) and 120 beam elements (vessels). The simulation employing this model was running stably in real-time: refresh rate of 25 FPS was achieved on PC having Intel i7 M620 $2.76 \mathrm{GHz}$ processor with $960 \times 540$ images acquired from the DaVinci Robot provided by Intuitive Surgical $\AA$. According to [31] the parameters of the LucasKanade optical flow include the window size of $51 \times 51$ pixels and an inter-frame motion threshold of 20 pixels. We use the framework Sofa [42] for the simulation of the elastic behaviour of the heterogeneous liver model.

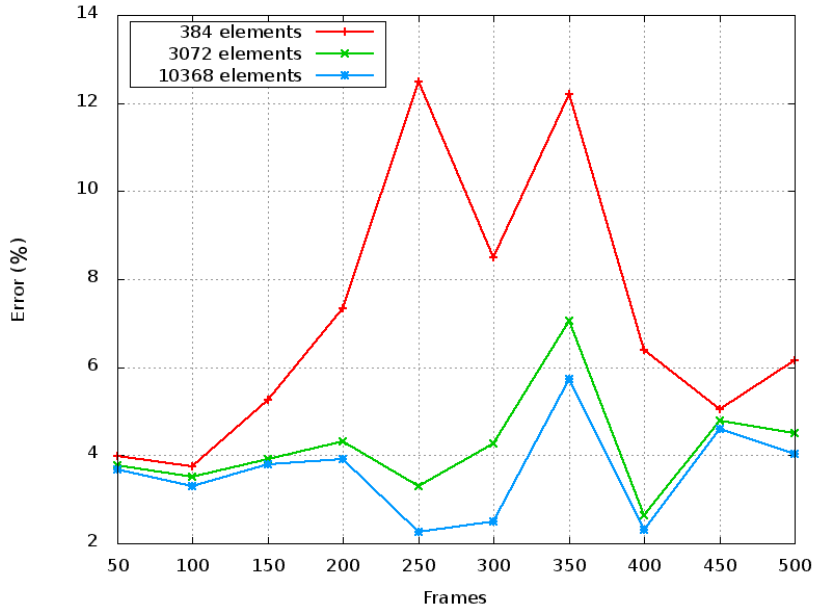

Fig. 14. Projection error of the overlaid mesh on the image with a variation on the number of element.

Fig. 15 displays four frames of the augmentation computed during the simulation and shows a good visual match between the images and the liver mesh.

Since no quantitative results can be obtained on in vivo data about the internal tumor position, we propose to compute the projection error of the overlaid mesh on the laparoscopic image. To do so, we measure a distance between the overlaid mesh contour and the liver contour both extracted manually. The Fig. 14 illustrates these distances and shows that increasing the number of tetrahedral elements reduces significantly the superimposition errors.

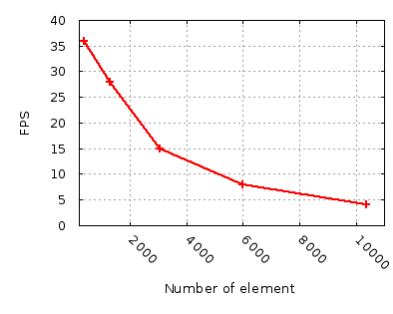

(a)

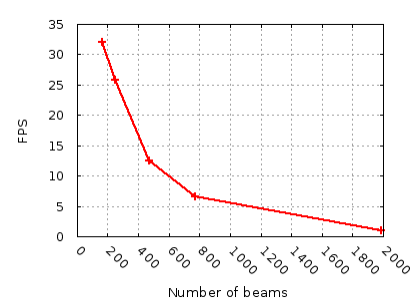

(b)
Fig. 16. Performance of the simulation w.r.t. the number of elements: (a): different density of parenchyma meshes (while number of beams equals to 164), (b): different density of beam elements (while the same tetrahedral mesh with 3072 elements used).

The computational cost of the simulation is given by several factors. First, due to the heterogeneity and anisotropy introduced by the vascular structures, iterative solvers (such as conjugate gradients) cannot be used due the to convergence issues. Therefore, a direct solver (such as LDL-decomposition) is necessary to solve the system in each time step. However, the time needed to compute the LDL decomposition increases rapidly with the number of mechanical degrees of freedom of the model, which is given by the number of tetrahedral elements representing the parenchyma. Further, the number of beam elements representing the vessels affects the performance as well 

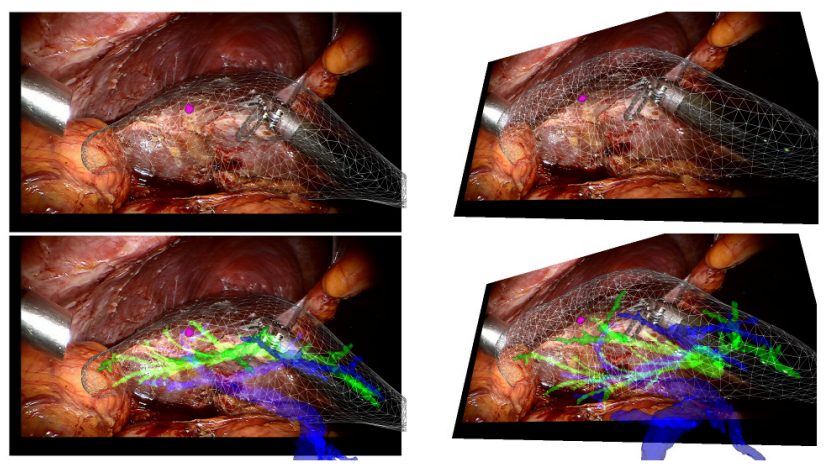

(a)
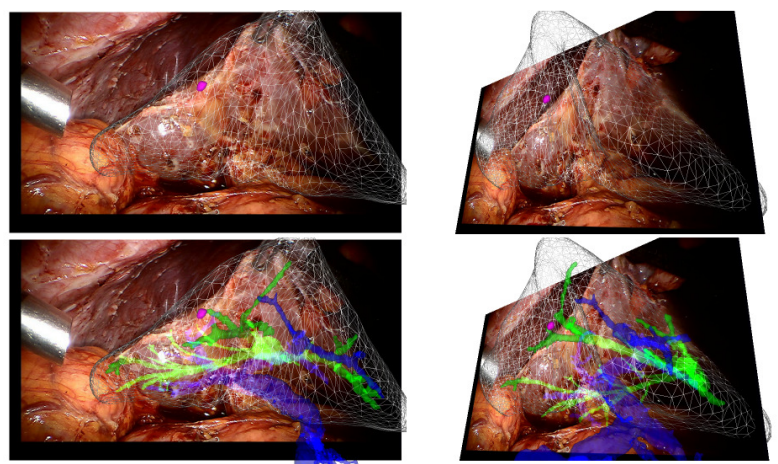

(c)
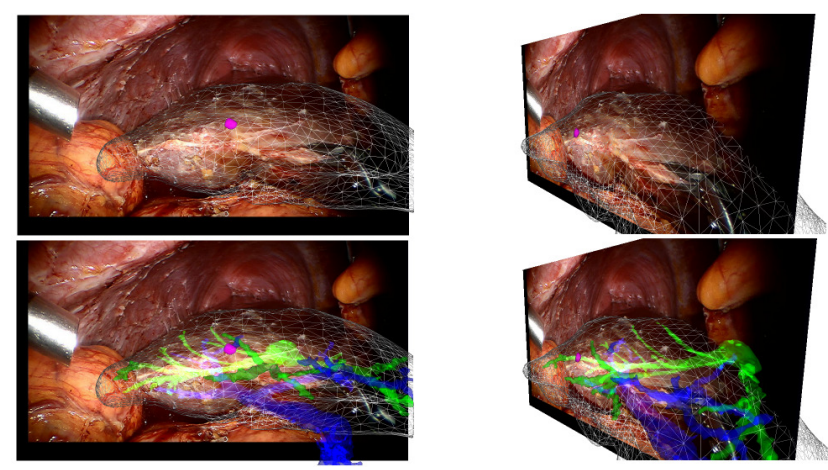

(b)
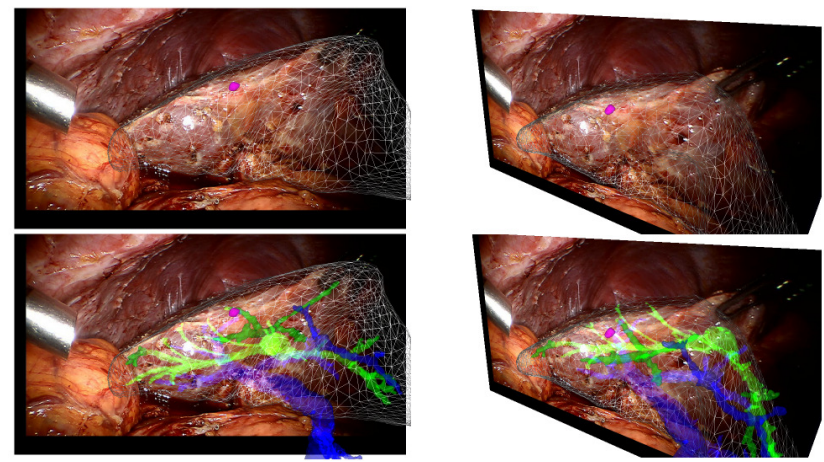

(d)

Fig. 15. A sequence of images showing, in different views, the superimposition of the real-time biomechanical model onto the human liver undergoing deformation due to surgical instrument interaction during MIS. The liver is represented in wire-frame, the tumor in purple, the hepatic vein is shown in blue and the portal vein in green.

due to the computation of mapping which is calculated in each step of the simulation. Fig. 16 shows the relation between the number of tetrahedral and beam elements and the refresh rate of the simulation. In order to achieve a realtime performance, we use the mesh having 3072 tetrahedra and 164 beams.

The stiffness corresponding to the external stretching force (parameter $k$ in Eq. 5 has to be chosen very carefully. We determined the value empirically; it was set to be an order of magnitude higher than the Young's modulus of the parenchyma in order to fit correctly the tracked points. A higher stiffness may unbalance the minimization equation as illustrated in Fig. 17.
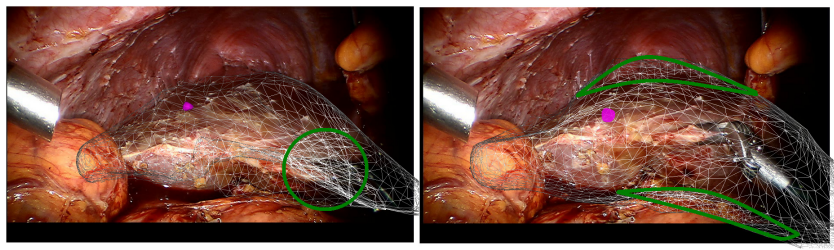

Fig. 17. Failed cases: external stretching forces with high stiffness may unbalance the minimization equation and forces the model to fit the tracked points that may be poorly estimated. Incorrectly registered areas are highlighted in green.

\section{LIMITATIONS AND DISCUSSION}

Both phantom and in vivo data present good results in term of surface registration and localisation of internal tumors. With an estimation of the position below the actual clinical margin and a computation time compatible with the realtime visual feedback, we believe that our method is a very promising solution. Yet, a few limitations remain to be solved. Several disturbances may occur during the tracking stage due to the outliers. One way to reduce the impact of the outliers, is the labelling of the intra-abdominal scene by segmenting the liver, the surrounding tissues and the surgical tools to isolate the liver surface.

Another limitation concerning the practical implementation of the method in the clinical environment is given by the initial registration, which is done manually. As stated before, substantial improvement of this part of the work flow is beyond the scope of this paper since the solution is quite complex as the underlying problem is ill-posed due to the difficult extraction of salient geometrical and anatomical landmarks from the patient data.

From a simulation point of view, we believe that the use of a real-time heterogeneous biomechanical model permits to accurately reproduce the behaviour of the liver undergoing large deformations. However, we are aware of the fact that a better definition of the boundary conditions is needed to capture its motion with increased accuracy. We consider addressing this issue already in the initial registration phase. 
Finally, a correct estimation of the mechanical properties is another key aspect that is to be improved, as these parameters are known to vary significantly in liver. We believe that integrating the elastography data recorded preoperatively could substantially increase the accuracy of the model.

\section{Conclusion}

The work presented in this paper deals with accurate localisation of internal structures such as tumours which superimposed on the organ surface during minimally invasive surgery. To this end, we developed an image-guided biomechanical model that is able to capture the complex deformations undertaken by the liver during the surgery. A complete experimental bench capable to reproduce as closely as possible a typical scenario encountered during liver tumor resection was designed. Promising results were obtained using in-vivo human liver data and a liver phantom, with internal structures localisation errors below the safety margins actually preserved during the interventions.

Future work will address the ill-posed issue of initialization and evaluate its impact on the overall process. A better definition of the external forces generated from the tracking in the stretching energy minimization will also be sought while the visualization of the vascular network overlaid in the laparoscopic view will be improved to ensure a better depth perception.

\section{REFERENCES}

[1] S. Nicolau, L. Soler, D. Mutter, and J. Marescaux, "Augmented reality in laparoscopic surgical oncology," Surgical Oncology, vol. 20, no. 3, pp. $189-201,2011$.

[2] A. Mitiche and P. Bouthemy, "Computation and analysis of image motion: a synopsis of current problems and methods," Int. J. Comput. Vision, vol. 19, no. 1, pp. 29-55, July 1996.

[3] A. Bartoli and A. Zisserman, "Direct estimation of non-rigid registration," in In British Machine Vision Conference, 2004

[4] J. Pilet, V. Lepetit, and P. Fua, "Fast non-rigid surface detection, registration and realistic augmentation," Int. J. Comput. Vision, vol. 76, no. 2, pp. 109-122, Feb. 2008.

[5] J. Zhu and M. R. Lyu, "Progressive finite newton approach to realtime nonrigid surface detection," in Computer Vision and Pattern Recognition, 2007. IEEE Conference on. IEEE, 2007, pp. 1-8.

[6] V. Gay-Bellile, A. Bartoli, and P. Sayd, "Direct estimation of nonrigid registrations with image-based self-occlusion reasoning," IEEE Trans. Pattern Anal. Mach. Intell., vol. 32, no. 1, pp. 87-104.

[7] A. Hilsmann, D. C. Schneider, and P. Eisert, "Realistic cloth augmentation in single view video under occlusions," Comput. Graph., vol. 34, no. 5, pp. 567-574, Oct. 2010.

[8] M. Salzmann, J. Pilet, S. Ilic, and P. Fua, "Surface deformation models for nonrigid 3d shape recovery," IEEE Trans. Pattern Anal. Mach. Intell., vol. 29, no. 8, pp. 1481-1487, Aug. 2007.

[9] S. Shen, W. Shi, and Y. Liu, "Monocular 3-d tracking of inextensible deformable surfaces under 12-norm," Trans. Img. Proc., vol. 19, no. 2, pp. 512-521, Feb. 2010.

[10] T. Hayashi, F. de Sorbier, and H. Saito, "Texture overlay onto non-rigid surface using commodity depth camera," in International Conference on Computer Vision Theory and Applications, 2012, pp. $66-71$.

[11] A. Agudo, B. Calvo, and J. M. M. Montiel, "Finite element based sequential bayesian non-rigid structure from motion," in CVPR, 2012, pp. 1418-1425.

[12] S. Wuhrer, J. Lang, and C. Shu, "Tracking complete deformable objects with finite elements." in 3DIMPVT. IEEE, 2012, pp. 1-8.
[13] A. Malti, R. Hartley, A. Bartoli, and J.-H. Kim, "Monocular template-based $3 \mathrm{~d}$ reconstruction of extensible surfaces with local linear elasticity," in Computer Vision and Pattern Recognition, 2013 IEEE Conference on, 2013, pp. 1522-1529.

[14] D. Teber, S. Guven, T. Simpfendrfer, M. Baumhauer, E. O. Gven, F. Yencilek, A. S. Gzen, and J. Rassweiler, "Augmented reality: A new tool to improve surgical accuracy during laparoscopic partial nephrectomy? preliminary in vitro and in vivo results," European Urology, vol. 56, no. 2, pp. 332 - 338, 2009.

[15] M. Figl, D. Rueckert, D. Hawkes, R. Casula, M. Hu, O. Pedro, D. P. Zhang, G. Penney, F. Bello, and P. Edwards, "Image guidance for robotic minimally invasive coronary artery bypass," Computerized Medical Imaging and Graphics, vol. 34, no. 1, pp. 61-68, 2010.

[16] L.-M. Su, B. P. Vagvolgyi, R. Agarwal, C. E. Reiley, R. H. Taylor, and G. D. Hager, "Augmented reality during robot-assisted laparoscopic partial nephrectomy: Toward real-time 3d-ct to stereoscopic video registration," Urology, vol. 73, no. 4, pp. 896 - 900, 2009.

[17] J.-H. Kim, A. Bartoli, T. Collins, and R. Hartley, "Tracking by detection for interactive image augmentation in laparoscopy," in Proceedings of the 5th international conference on Biomedical Image Registration, ser. WBIR'12. Springer-Verlag, 2012, pp. 246-255.

[18] G. Puerto-Souza and G. Mariottini, "Toward long-term and accurate augmented-reality display for minimally-invasive surgery," in Robotics and Automation (ICRA), 2013 IEEE International Conference on, 2013, pp. 5384-5389.

[19] M. Sermesant, C. Forest, X. Pennec, H. Delingette, and N. Ayache, "Deformable biomechanical models: Application to 4D cardiac image analysis," Medical Image Analysis, vol. 7, no. 4, pp. 475-488, 2003.

[20] T. Shen, H. Li, and X. Huang, "Active volume models for medical image segmentation," IEEE Trans. Med. Imaging, vol. 30, no. 3, pp. 774-791, 2011.

[21] S. Suwelack, S. Rhl, S. Bodenstedt, D. Reichard, R. Dillmann, T. dos Santos, L. Maier-Hein, M. Wagner, J. Wnscher, H. Kenngott, B. P. Mller, and S. Speidel, "Physics-based shape matching for intraoperative image guidance," Medical Physics, vol. 41, no. 11, pp.,- 2014

[22] P. Pratt, D. Stoyanov, M. Visentini-Scarzanella, and G.-Z. Yang, "Dynamic guidance for robotic surgery using image- constrained biomechanical models," in Proceedings of the 13th MICCAI: Part I, ser. MICCAI'10. Springer-Verlag, 2010, pp. 77-85.

[23] J. Schaerer, C. Casta, J. Pousin, and P. Clarysse, "A dynamic elastic model for segmentation and tracking of the heart in $\mathrm{mr}$ image sequences," Medical Image Analysis, vol. 14, no. 6, pp. 738 - 749, 2010.

[24] N. Haouchine, J. Dequidt, E. Kerrien, M.-O. Berger, and S. Cotin, "Physics-based Augmented Reality for 3D Deformable Object," in VRIPHYS - Virtual Reality Interaction and Physical Simulation, Darmstadt, Germany, Dec. 2012, pp. 31-38.

[25] N. Haouchine, J. Dequidt, M.-O. Berger, and S. Cotin, "Deformationbased Augmented Reality for Hepatic Surgery," in MMVR 20, San Diego, USA, 2013, pp. 182-188.

[26] N. Haouchine, J. Dequidt, I. Peterlik, E. Kerrien, M.-O. Berger, and S. Cotin, "Image-guided simulation of heterogeneous tissue deformation for augmented reality during hepatic surgery." in ISMAR 2013, pp. 199-208.

[27] L. Maier-Hein, A. Groch, A. Bartoli, S. Bodenstedt, G. Boissonnat, P.-L. Chang, N. Clancy, D. Elson, S. Haase, E. Heim, J. Hornegger, P. Jannin, H. Kenngott, T. Kilgus, B. Muller-Stich, D. Oladokun, S. Rohl, T. dos Santos, H.-P. Schlemmer, A. Seitel, S. Speidel, M. Wagner, and D. Stoyanov, "Comparative validation of singleshot optical techniques for laparoscopic 3-d surface reconstruction," Medical Imaging, IEEE Transactions on, vol. 33, no. 10, pp. 19131930, Oct 2014

[28] L. Maier-Hein, P. Mountney, A. Bartoli, H. Elhawary, D. Elson, A. Groch, A. Kolb, M. Rodrigues, J. Sorger, S. Speidel, and D. Stoyanov, "Optical techniques for $3 \mathrm{~d}$ surface reconstruction in computer-assisted laparoscopic surgery," Medical Image Analysis, vol. 17, no. 8, pp. $974-996,2013$.

[29] H. Bay, A. Ess, T. Tuytelaars, and L. Van Gool, "Speeded-up robust features (SURF)," Comput. Vis. Image Underst., vol. 110, no. 3, pp. 346-359, June 2008.

[30] B. D. Lucas and T. Kanade, "An iterative image registration technique with an application to stereo vision," in Proceedings of the 7th international joint conference on Artificial intelligence - Volume 2, ser. IJCAI'81. San Francisco, CA, USA: Morgan Kaufmann Publishers Inc., 1981, pp. 674-679. 
[31] H. Elhawary and A. Popovic, "Robust feature tracking on the beating heart for a robotic-guided endoscope." Int J Med Robot., pp. 459 468, Oct. 2010.

[32] N. Haouchine, J. Dequidt, I. Peterlik, E. Kerrien, M.-O. Berger, and S. Cotin, "Towards an accurate tracking of liver tumors for augmented reality in robotic assisted surgery," in Robotics and Automation (ICRA), 2014 IEEE International Conference on, 2014, pp. $4121-4126$.

[33] Z. Zhang, "A flexible new technique for camera calibration," IEEE Trans. Pattern Anal. Mach. Intell., vol. 22, no. 11, pp. 1330-1334.

[34] A. E. Kerdok, M. P. Ottensmeyer, and R. D. Howe, "Effects of perfusion on the viscoelastic characteristics of liver," Journal of Biomechanics, vol. 39, pp. 2221-2231, 2006.

[35] Z. Gao, T. Kim, D. L. James, and J. P. Desai, "Semi-automated softtissue acquisition and modeling forsurgical simulation," in Proceedings of the fifth annual IEEE international conference on Automation science and engineering, ser. CASE'09. Piscataway, NJ, USA: IEEE Press, 2009, pp. 268-273.

[36] C. Felippa and B. Haugen, "A unified formulation of small-strain corotational finite elements: I. theory," Computer Methods in Applied Mechanics and Engineering, vol. 194, no. 21, pp. 2285-2335, 2005.

[37] M. Nesme, Y. Payan, and F. Faure, "Efficient, physically plausible finite elements," in Eurographics 2005, Short papers, August, 2005, J. Dingliana and F. Ganovelli, Eds., Trinity College, Dublin, Irlande, 2005.

[38] S. Marchesseau, T. Heimann, S. Chatelin, R. Willinger, and H. Delingette, "Multiplicative jacobian energy decomposition method for fast porous visco-hyperelastic soft tissue model," in MICCAI 2010, ser. LNCS, 2010, vol. 6361, pp. 235-242.

[39] I. Peterlik, C. Duriez, and S. Cotin, "Modeling and real-time simulation of a vascularized liver tissue," in Proceedings of the 15th international conference on MICCAI - Volume Part I, ser. MICCAI'12. Springer-Verlag, 2012, pp. 50-57.

[40] C. Duriez, S. Cotin, J. Lenoir, and P. Neumann, "New approaches to catheter navigation for interventional radiology simulation 1," Computer Aided Surgery, vol. 11, no. 6, pp. 300-308, 2006.

[41] J. S. Przemieniecki, Theory of Matrix Structural Analysis, 1985, reprint of McGraw Hill, 1968.

[42] F. Faure, C. Duriez, H. Delingette, J. Allard, B. Gilles, S. Marchesseau, H. Talbot, H. Courtecuisse, G. Bousquet, I. Peterlik, and S. Cotin, "SOFA: A Multi-Model Framework for Interactive Physical Simulation," in Soft Tissue Biomechanical Modeling for Computer Assisted Surgery, ser. Studies in Mechanobiology, Tissue Engineering and Biomaterials. Springer Berlin Heidelberg, June 2012, vol. 11, pp. 283-321. [Online]. Available: http://www.sofaframework.com

[43] S. Umale, S. Chatelin, N. Bourdet, C. Deck, M. Diana, P. Dhumane, L. Soler, J. Marescaux, and R. Willinger, "Experimental in vitro mechanical characterization of porcine glisson's capsule and hepatic veins," Journal of Biomechanics, vol. 44, no. 9, 2011.

[44] F. Sanchez-Margallo, J. Moyano-Cuevas, R. Latorre, J. Maestre, L. Correa, J. Pagador, L. Sanchez-Peralta, J. Sanchez-Margallo, and J. Uson-Gargallo, "Anatomical changes due to pneumoperitoneum analyzed by mri: an experimental study in pigs," Surgical and Radiologic Anatomy, vol. 33, no. 5, pp. 389-396, 2011.

[45] A. Kerdok, S. Cotin, M. Ottensmeyer, A. Galea, R. Howe, and S. Dawson, "Truth cube : Establishing physical standards for real time soft tissue simulation," Medical Image Analysis, vol. 7, no. 3, pp. $283-291,2003$.

[46] T. Mala, B. Edwin, I. Gladhaug, E. Fosse, O. Sreide, A. Bergan, and $\mathrm{O}$. Mathisen, "A comparative study of the short-term outcome following open and laparoscopic liver resection of colorectal metastases," Surgical Endoscopy And Other Interventional Techniques, vol. 16, no. 7, pp. 1059-1063, 2002.

[47] H. Yamada, Strength of biological materials. Baltimore: The williams and Wilkins Company, 1970.

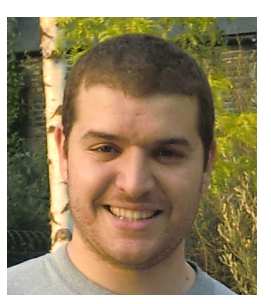

Nazim Haouchine graduated from the University of Science and Technology of Algiers (USTHB), Algeria, in 2010, and received an Master degree in computer science from the University of Lille 1, Lille, France in 2011. He is now a PhD student at INRIA with Shacra and Magrit groups. His research interests are in augmented reality, computer vision, medical imaging, image-guided simulation for surgery and clinical validations.

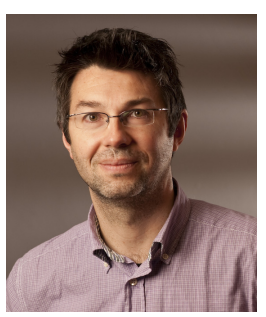

Stephane Cotin joined INRIA in 2007 to hold a position of Research Director. Since January 2010 he is also leader of the SHACRA group, a multidisciplinary team of scientists involved in the field on medical simulation. Before joining INRIA, he was Research Lead for the Medical Simulation Group at CIMIT (Boston, Ma). His main research interests are in physics-based simulation, real-time simulation of soft-tissue deformations, and medical applications of this research.

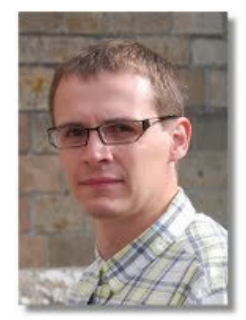

Igor Peterlik received the master and $\mathrm{PhD}$ degrees in informatics from Masaryk University, Czech Republic (2009). As postdoctoral fellow at INRIA Lille Nord Europe, he participated in European project PASSPORT (2009-2011). In 2012, he was granted a post-doctoral fellowship at University of British Columbia, Vancouver, Canada (2011 2012). He continued his research at Institut Hospitalo-Universitaire, Strasbourg, France (2012-2013). Currently, he is with the Institute of Computer Science, Masaryk University, Czech Republic. His research interests include soft-tissue modeling, real-time interaction with deformable objects, haptic rendering, image processing and parallel and distributed computing.

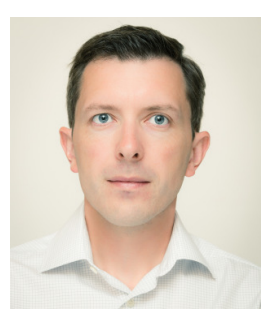

Jérémie Dequidt completed a Ph.D. thesis in Computer Science at the University of Lille 1 in 2005. As a post-doctoral fellow, he joined the SimGroup at CIMIT (Boston, $\mathrm{MA}$ ) and then INRIA Alcove team, working on interventional radiology simulations. Since september 2008, he is an Assistant Professor in Computer Science at University of Lille 1 . He also is a member of the Inria researchteam Shacra. His research interests focus on collision detection and response, mechani$\mathrm{cal} /$ geometric/adaptive modeling and experimental validations.

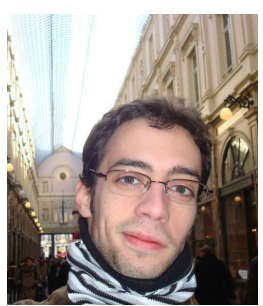

Mario Sanz Lopez received a master degree in telecommunications and electronic systems engineering from the University of Alcalà de Henares in Spain. Since 2012 he works as design engineer for INRIA, Lille. His interests focus on prototype design of new sensors and validation protocols concerning medical simulation and human interfaces for virtual reality environments, demonstrations and showrooms.

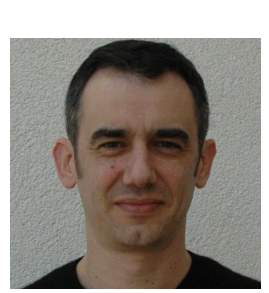

Erwan Kerrien graduated from the Ecole Nationale Supérieure des Télécommunications, Paris, France, in 1994, and received a $\mathrm{PhD}$ degree in computer science from the Institut National Polytechnique de Lorraine, Nancy, France in 2000. He is now a researcher at INRIA Nancy Grand-Est in the Loria Lab, Magrit group. His research interests are in computer vision and medical imaging, in particular multimodal imaging, augmented reality and image-driven simulation for interventional radiology and minimally invasive surgery.

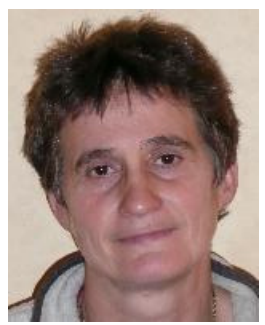

Marie-Odile Berger is presently research scientist at INRIA in computer vision. She is "agregée de mathematiques" and received a PhD in computer science from University Henri Poincaré at Nancy, France. She is currently the head of the computer vision group Magrit at INRIA Nancy Grand est. Her research interests include visual reconstruction, augmented reality, medical imaging, analysis of video sequences. 